\title{
Mutual synchronization in ensembles of globally coupled neural networks
}

\author{
D. H. Zanette* and A. S. Mikhailov \\ Fritz-Haber-Institut der Max-Planck-Gesellschaft, Faradayweg 4-6, 14195 Berlin, Dahlem, Germany
}

(Received 17 November 1997; revised manuscript received 26 January 1998)

\begin{abstract}
The collective dynamics in globally coupled ensembles of identical neural networks with random asymmetric synaptic connections is investigated. We find that this system shows a spontaneous synchronization transition, i.e., networks with synchronous activity patterns appear in the ensemble when the coupling intensity exceeds a threshold. Under further increase of the coupling intensity, the entire ensemble breaks down into a number of coherent clusters, until complete mutual synchronization is eventually established. [S1063-651X(98)02907-9]

PACS number(s): 87.10. $+\mathrm{e}, 87.22 . \mathrm{Jb}, 05.45 .+\mathrm{b}, 89.70 .+\mathrm{c}$
\end{abstract}

Synchronization phenomena in populations of globally coupled dynamical systems are a subject of intensive theoretical research. Since the pioneering study by Winfree [1], much attention has been attracted to investigations of large oscillator populations (see [2-4]). However, it is getting increasingly clear that synchronization does not represent a special feature of oscillator systems. For instance, recent studies have shown that similar behavior is observed in systems formed by globally coupled Hamiltonian [5] or bistable [6] elements. Moreover, it is also known that mutual synchronization is possible [7] in populations of coupled chaotic dynamical systems, such as logistic maps [8] or Rössler oscillators [9]. A detailed study of the synchronization transition in large populations of stochastic globally coupled systems has recently been performed [10].

The importance of synchronization for functioning of biological systems has been extensively discussed $[1,3,8]$. It has been emphasized that these effects should play a significant role in operation of the brain (see, e.g., [11]). Indeed, theoretical investigations show that mutual synchronization easily develops in populations of globally coupled individual neurons [11-13]. Examining the brain functions, one can, however, note that besides this strong kind of synchronization, resulting in identical states of all neurons in a uniform population, more subtle forms of synchronization should be present. The brain is essentially a system of interacting neural networks and the activity patterns of different networks may perhaps become synchronized while retaining their complex spatiotemporal dynamics. This puts forward a general theoretical problem of mutual synchronization in ensembles of coupled neural networks [11].

In the present paper this problem is addressed by studying a simple model system where the neurons are represented by dynamical McCulloch-Pitts elements [14]. A network is formed by such elements linked through activatory or inhibitory connections of varying weights. When asymmetric connection weights are chosen, such a network would generally exhibit complex spatiotemporal oscillations. We take an ensemble of identical networks that are linked together by in-

\footnotetext{
*Permanent address: Consejo Nacional de Investigaciones Científicas y Técnicas, Centro Atómico Bariloche and Instituto Balseiro, 8400 Bariloche, Argentina.
}

troducing additional global cross-network interactions between neurons occupying equivalent positions in different networks of the ensemble. The simulations reveal that the ensemble can easily undergo a spontaneous synchronization transition. In the fully synchronous regime, all networks are characterized by the same complex spatiotemporal activity pattern of neurons. At lower intensities of the cross-network coupling, the ensemble breaks into several coherent clusters.

We consider ensembles made of $N$ identical neural networks each consisting of $K$ neurons. The collective dynamics of an ensemble is described by the following algorithm: At time $t+1$, the activity $x_{k}^{i}$ of a neuron $k=1, \ldots, K$ belonging to a network $i=1, \ldots, N$ is

$$
x_{k}^{i}(t+1)=(1-\varepsilon) \Theta\left(h_{k}^{i}\right)+\varepsilon \Theta\left(\sum_{j=1}^{N} h_{k}^{j}\right) \text {, }
$$

where $h_{k}^{i}=\sum_{l=1}^{K} J_{k l} x_{l}^{i}(t)$ is the signal arriving at this neuron at time $t$ from all other elements of the same network, $J_{k l}$ are the connection weights (the same for all networks), and $\Theta(z)$ is a sigmoidal function.

The two terms on the right-hand side of Eq. (1) have a clear interpretation. The first of them represents the individual response of a neuron to the total signal received from other elements in its own network. The second term depends on the global signal obtained by summation of individual signals received by neurons occupying the same positions in all networks of the ensemble (and hence it corresponds to global cross-network interactions). The parameter $\varepsilon$ specifies the strength of global coupling. When global coupling is absent $(\varepsilon=0)$, the networks forming the ensemble are independent. On the other hand, at $\varepsilon=1$ the first term vanishes and the states of respective neurons in all networks must be identical, since they are determined by the same global signal. For $0<\varepsilon<1$, the ensemble dynamics is governed by an interplay between local coupling inside the networks and global coupling across them.

Our analysis is based on numerical investigations. As the first step, we set up the connection weights between neurons in the individual network. Each of the connection weights $J_{k l}$ between neurons is chosen at random with equal probability from the interval between -1 to 1 . The weights of forward and reverse connections are independently selected, and 
(a)

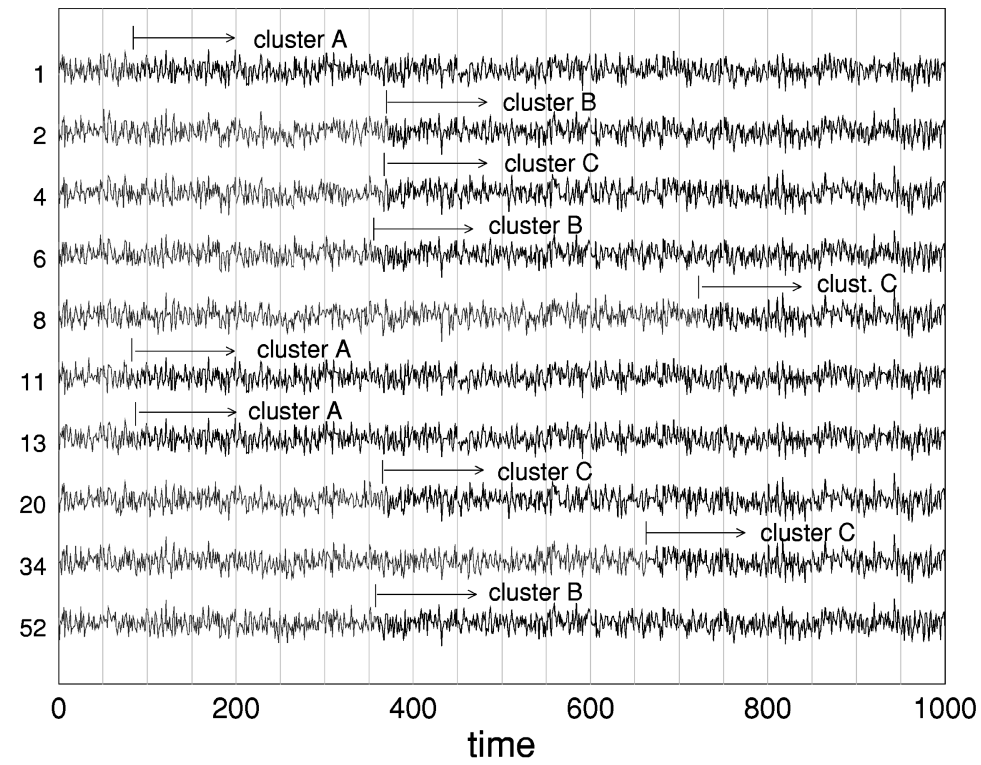

(b)

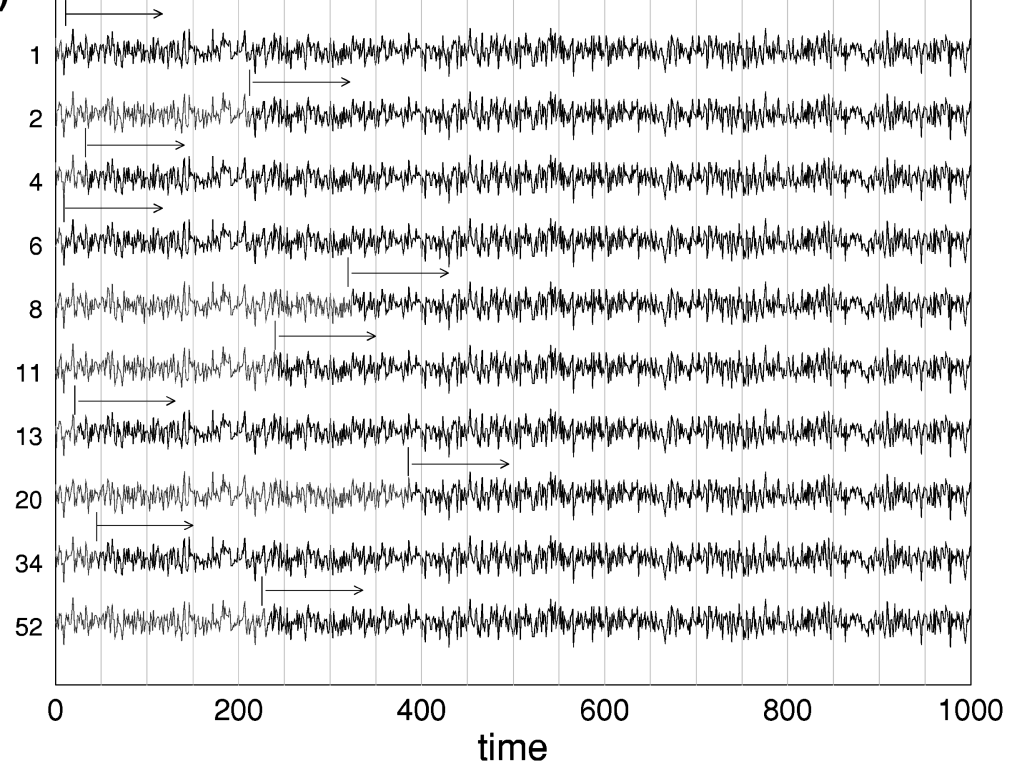

FIG. 1. Time-dependent integral activity of ten selected networks $(K=50)$ in an ensemble of size $N=100$ for different intensities of global coupling, corresponding to (a) clustering ( $\varepsilon$ $=0.35)$ and (b) full synchronization $(\varepsilon=0.5)$. Synchronization of each signal begins at the corresponding bar. In (a), clusters are identified by different letters. therefore $J_{k l} \neq J_{l k}$. The sigmoidal function in Eq. (1) has been taken as $\Theta(z)=[1+\tanh (\beta z)] / 2$, with $\beta=10$. Most of the simulations have been performed for ensembles of $N$ $=100$ identical networks, each consisting of $K=50$ neurons. The connection weights have remained fixed within the entire series of simulations with varying global coupling intensity. The initial conditions for all neurons in all networks in each simulation have been randomly chosen.

Since subsequent states of all neurons in all networks are recorded, and each simulation yields a large volume of data that should be further analyzed in order to detect coherence in the collective activity of the ensemble. An important property is the integral time-dependent activity $u_{i}(t)$ $=\sum_{k=1}^{K} x_{k}^{i}(t)$ of each network $i=1, \ldots, N$ in the ensemble. If global coupling is absent or very weak, the networks are independent and, since the initial conditions are various for different networks, their activity patterns are not correlated. Therefore, the integral signals $u_{i}(t)$ generated by different networks in the ensemble would be asynchronous. On the other hand, if synchronization of the network activity has taken place, the activity patterns of some networks are identical and their integral time-dependendent signals will also coincide.

Figure 1 shows typical integral signals generated by networks when global coupling is relatively strong. Though the integral signals of the networks are at first not correlated, starting from a certain moment, some of the networks in the ensemble begin to generate identical (up to the computer precision) signals, indicating the onset of synchronization in the system. When $\varepsilon=0.35$ [Fig. 1(a)], the entire ensemble breaks down into several synchronous clusters. At a higher intensity of global coupling $[\varepsilon=0.5$, Fig. 1(b)], the activity of all networks in the ensemble is synchronous.

We have performed a special numerical investigation of the clustering regime shown in Fig. 1(a). By continuing the simulation over further $10^{5}$ time steps, no relaxation to synchronous oscillations has been found. Three clusters, formed by time $t=1000$, persisted in the system. Moreover, clustering has always been observed when we performed 100 independent runs with randomly chosen initial conditions. It 


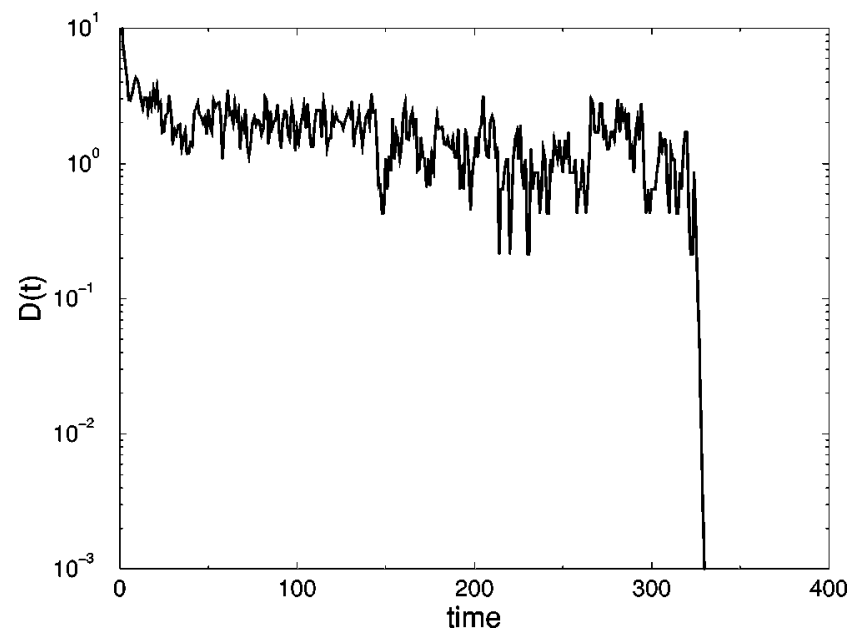

FIG. 2. Dispersion of the activity patterns of all networks in the ensemble as function of time under synchronization conditions $(\varepsilon$ $=0.5, \quad N=100, \quad K=50)$.

should be noted that stable partially synchronous states, where the entire population of globally coupled dynamical systems forms a number of coherent clusters, have previously been reported for relatively simple systems, such as logistic maps [8] or chaotic Rössler oscillators [10]. Our present results indicate that both full synchronization and clustering are also possible in ensembles whose individual elements represent complex dynamical systems.

The degree of synchronization in the ensemble dynamics can be characterized by the dispersion of activity patterns, defined as $D(t)=N^{-1} \sum_{i=1}^{N} \Sigma_{k=1}^{K}\left[x_{k}^{i}(t)-\bar{x}_{k}(t)\right]^{2}$ where $\bar{x}_{k}(t)=N^{-1} \sum_{j=1}^{N} x_{k}^{j}(t)$ is the average activity of neurons occupying the position $k$ in all networks of the ensemble at time $t$. Fig. 2 shows, on a logarithmic scale, how this property evolves in time in a typical simulation at a fixed intensity $\varepsilon=0.5$ of global coupling.

To explain this time dependence of $D(t)$, the process by which full synchronization is established in the ensemble should be analyzed. As seen in Fig. 1(b), synchronization begins with the formation of a coherent nucleus consisting of a few networks. This nucleus grows by an aggregation process, where further networks are subsequently added to it, i.e., become entrained. While nonentrained networks remain, the dispersion $D(t)$ remains relatively large, though it gradually decreases with time. When the last network has approached the coherent cluster, it gets strongly attracted and its distance from the cluster begins to exponentially decrease. This rapid decrease is reflected in the final linear steep fall seen in Fig. 2.

Though the dispersion serves as a good indicator of full synchronization, it is not sensitive to partial synchronization and formation of coherent clusters in the ensemble. To analyze clustering, a different statistical method has therefore been employed that involved calculation of pair distances between activity patterns of all networks. The pair distance between the activity patterns of two networks $i$ and $j$ is defined as $d_{i j}=\left[\sum_{k=1}^{K}\left(x_{k}^{i}-x_{k}^{j}\right)^{2}\right]^{1 / 2}$. By counting the number of network pairs in the whole ensemble that have at a given time the distances lying within subsequent equal intervals, a histogram of distribution over pair distances can be con-

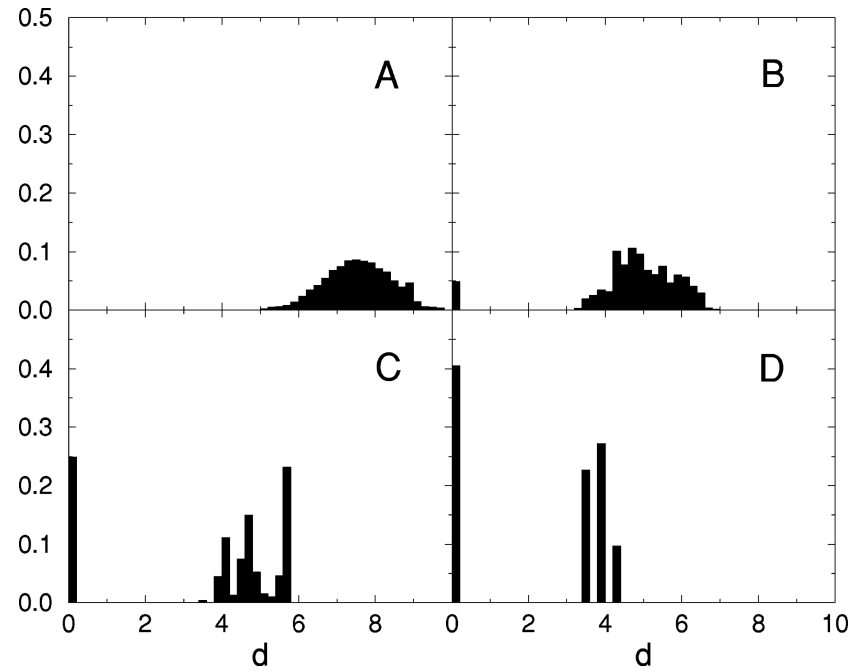

FIG. 3. Histograms of distributions over pair distances $d$ between activity patterns of all networks in the ensemble $(N$ $=100, K=50$ ) for various intensities of global coupling corresponding to the asynchronous regime (a) $\varepsilon=0.15$, and different partially synchronous regimes (b) $\varepsilon=0.28$, (c) $\varepsilon=0.34$, and (d) $\varepsilon$ $=0.35$.

structed. Figure 3 shows these normalized histograms for several intensities of global coupling.

When global coupling is weak $[\varepsilon=0.15$, Fig. 3(a)], the histogram has a single smooth maximum at a typical distance between the activity patterns of noncorrelated networks. Increasing the coupling intensity, we find that above a certain critical point $\left(\varepsilon_{1} \approx 0.17\right)$ some pairs of networks in the ensemble have exactly the same activity patterns, so that the distance between them is zero. This corresponds to the presence of a peak at $d=0$ in the histogram shown in Fig. 3(b) for $\varepsilon=0.28$. When global coupling is further increased, the number of identical pairs grows $[\varepsilon=0.34$, Fig. 3(c)]. The synchronous networks are apparently organized into clusters. Indeed, several peaks are seen in this histogram. The peaks are located at pair distances between different clusters. However, besides these clusters the ensemble still has a number of networks with asynchronous activity. A slight increase of global coupling leads to the emergence of a definite cluster organization $[\varepsilon=0.35$, Fig. 3(d)]. In this case, every network belongs to one of a few synchronous clusters. As the coupling intensity grows, the number of clusters gets smaller, until full synchronization is established in the ensemble at $\varepsilon_{2} \approx 0.4$ (this final regime is not shown in Fig. 3).

We have repeated our simulations and statistical analysis for different random choices of connection weights in the networks and have observed basically the same sequence of changes leading to clustering and final synchronization in all studied cases, though the respective critical coupling intensities have been found to depend on the choice of connection weights. Moreover, essentially the same results have been obtained when ensembles consisting of larger networks of 100 neurons were studied and when other sigmoidal functions $\Theta(z)$ in the algorithm (1) were employed.

The considered ensemble can be viewed as a structure made of horizontal layers that represent individual networks. In addition to lateral interactions within its own layer, the neurons are involved in global vertical interactions across the 
layers. In the model (1) we have assumed that such vertical cross-network interactions span the system through each neuron in each of the networks. In some of the potential applications, it would be, however, more realistic to assume that only a certain fraction of neurons is taking part in the global cross-network interactions. With this in mind, we have also studied a modification of the model (1) where only a randomly chosen subset of neurons in each of the networks is involved in global cross-network interactions. In this situation the collective ensemble dynamics is defined by the algorithm $x_{k}^{i}(t+1)=\left(1-\varepsilon \xi_{k}\right) \Theta\left(h_{k}^{i}\right)+\varepsilon \xi_{k} \Theta\left(\sum_{j=1}^{N} h_{k}^{j}\right)$, where $\xi_{k}$ represent random variables taking the values 0 or 1 with probability $1-p$ and $p$, respectively. Thus, only $p K$ of neurons in any network are sensitive to global signals.

We have performed a large number of numerical simulations of this modified system following the same procedures as for the original model (1). New initial conditions have been independently randomly chosen in each of the simulations. Based on this numerical investigation, an approximate synchronization diagram shown in Fig. 4 has been constructed. Full synchronization is found inside the dark-grey region and partial synchronization (with clusters of coherent networks) is observed in the light-grey area. Note that in the latter case either all networks belong to such coherent clusters or some of the networks are still not entrained and have asynchronous activity (cf. Fig. 3). Examining the diagram in Fig. 4, one can see that the synchronization persists even when a significant fraction of cross-network connections is missing. Indeed, partial synchronization can still be observed even when only about $10 \%$ of global cross-network connections remains in the ensemble.

Synchronization of network activity and formation of coherent network clusters have been found in our study under a random choice of synaptic connections, and for networks and ensembles of various sizes. Moreover, such phenomena are also persisting when many randomly selected cross-network connections are erased. All this suggests that mutual synchronization of complex dynamic activity patterns may represent a generic property of globally coupled neural networks. Though we have studied in this paper only networks with random synaptic connections between neurons, similar

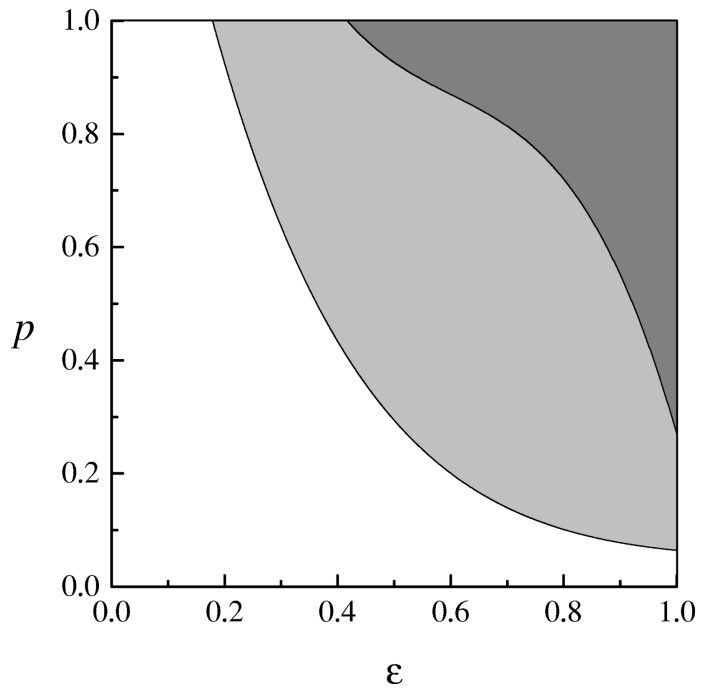

FIG. 4. Synchronization diagram in the parameter plane $(\varepsilon, p)$ for an ensemble of 100 networks consisting of 50 neurons with randomly chosen asymmetric synaptic connections. Full synchronization, with identical activity patterns of all networks, is found in the dark-grey area. Partial synchronization, defined by the presence of at least two networks with identical activity patterns, is observed in the light grey region.

synchronization properties should apparently be expected in situations where connection patterns are not arbitrary, but are rather designed in such a way that a neural network is able to perform certain operations of information processing or control the activity of motor units. Therefore, potential applications of this study may lie in the fields of multiagent robotics and distributed artificial intelligence.

The authors thank Professor B. Hess for stimulating discussions, and P. Stange for his help with the figures. D.H.Z. is grateful to Alexander von Humboldt-Stiftung and to the Fritz Haber Institute of the Max Planck Society for their hospitality during his stay in Berlin. Financial support from Fundación Antorchas, Argentina, is acknowledged.
[1] A. T. Winfree, The Geometry of Biological Time (Springer, Berlin, 1980).

[2] Y. Kuramoto, Chemical Oscillations, Waves and Turbulence (Springer, Berlin, 1984); H. Sakaguchi and Y. Kuramoto, Prog. Theor. Phys. 76, 576 (1986); N. Nakagawa and Y. Kuramoto, Physica D 75, 74 (1994).

[3] S. H. Strogatz, R. E. Mirollo, and P. C. Matthews, Phys. Rev. Lett. 68, 2730 (1992).

[4] V. Hakim and W.-J. Rappel, Phys. Rev. A 46, R7347 (1992)

[5] D. H. Zanette and A. S. Mikhailov, Phys. Lett. A 235, 135 (1997).

[6] D. H. Zanette, Phys. Rev. E 55, 5315 (1997).

[7] H. Fujisaka and T. Yamada, Prog. Theor. Phys. 69, 32 (1983).
[8] K. Kaneko, Physica D 23, 436 (1986); 37, 60 (1989); 54, 5 (1991).

[9] J. F. Heagy, T. L. Carrol, and L. M. Pecora, Phys. Rev. E 50, 1874 (1994)

[10] D. H. Zanette and A. S. Mikhailov, Phys. Rev. E 57, 276 (1998).

[11] H. D. Abarbanel, M. I. Rabinovich, A. Selverston, M. V. Bazhenov, R. Huerta, M. M. Sushchik, and L. L. Rubchinskii, Usp. Fiz. Nauk. 166, 363 (1996) [Phys. Usp. 39, 337 (1996)].

[12] H. Sompolinsky, D. Golomb, and D. Kleinfeld, Phys. Rev. A 43, 6990 (1991).

[13] D. Golomb, D. Hansel, B. Shraiman, and H. Sompolinsky, Phys. Rev. A 45, 3516 (1992).

[14] W. C. McCulloch and W. Pitts, Bull. Math. Biophys. 5, 115 (1943). 